\title{
La vaccinazione nel paziente con infezione da HIV: attuali raccomandazioni e strategie multidisciplinari per il raggiungimento dei soggetti suscettibili. L'esperienza della Liguria. Vaccination in HIV-infected patients: current recommendations and multidisciplinary strategy to reach susceptible subjects. The Ligurian experience.
}

Autore

per la corrispondenza:

Laura Sticchi

Department of Health Sciences (DiSSal),

University of Genoa

Via A. Pastore 1

16132 Genoa, Italy

Telephone:

+390105554651

Fax: +39010 505618

sticchi@unige.it

Keywords:

HIV, vaccination, guidelines

Potenziali conflitti

di interesse: Laura Sticchi

ha avuto rapporti con GSK,

Sanofi, Merck, Siemens,

Sequirus, Abbott, Novartis

JHA 2017; 2(3): 71-84

DOI: 10.19198/JHA31436

Laura Sticchi ${ }^{1,2}$, Antonio Di Biagio ${ }^{3}$, Mauro Giacomini ${ }^{4}$, Sergio Schiaffino ${ }^{5}$, Ilaria Barberis ${ }^{1}$, Valentino Tisa ${ }^{1}$, Federico Grammatico ${ }^{1}$, Giovanni Cenderello ${ }^{6}$, Barbara Giannini ${ }^{4}$, Valeria Faccio ${ }^{1}$, Giovanni Cassola ${ }^{6}$, Claudio Viscoli ${ }^{1,3}$, Giancarlo Icardi ${ }^{1,2}$

${ }^{1}$ Dipartimento di Scienze della Salute, Università degli Studi di Genova, Genova

${ }^{2}$ Unità Operativa Igiene, Ospedale Policlinico San Martino-IST, Genova

${ }^{3}$ Unità Operativa Clinica Malattie Infettive, Ospedale Policlinico San Martino-IST, Genova

${ }^{4}$ Dipartimento di Informatica, Bioingegneria, Robotica e Ingegneria dei Sistemi (DIBRIS), Università degli Studi di Genova, Genova

${ }^{5}$ Area dipartimentale Socio-Sanitaria, Azienda ligure sanitaria (Alisa), Genova

${ }^{6}$ Unità Operativa Clinica Malattie Infettive, Ente Ospedaliero Ospedali Galliera, Genova

Riassunto

Anche riell'era delle attuali efficaci terapie antiretrovirali di combinazione (CART), il controllo delle malattie infettive prevenibili mediante vaccinazione rappresenta un aspetto cruciale nei soggetti con infezione da HIV. Nonostante vi siano per loro specifiche indicazioni e la disponibilità di preparati sicuri ed efficaci, i dati della letteratura segnalano coperture vaccinali non soddisfacenti sia a livello nazionale sia internazionale.

II LIgurian Vaccination in HIV Program (LIV in HIV) è un'iniziativa su base regionale per la ricerca attiva, l'individuazione ed il raggiungimento dei soggetti suscettibili per le malattie prevenibili mediante vaccinazione. $E^{\prime}$ stato creato un percorso assistenziale condiviso, che ha coinvolto gli operatori dei centri vaccinali, dei centri di riferimento per la diagnosi e cura di questi pazienti, dei pediatri di libera scelta e dei medici di medicina generale.

Nell'ambito del LIV in HIV, è stato avviato il processo
Abstract

Even in the era of highly active antiretroviral therapy (HAART), HIV-infected subjects are at higher risk of complications from vaccine-preventable diseases than those uninfected.

Despite the current national and international guidelines strongly recommend that these patients should been immunized, the available data indicate sub-optimal coverage rates, partially due to the difficulty in identifying those susceptible to preventable infections.

In order to reach these subjects, the LIgurian Vaccination in HIV Program (LIV in HIV) aimed at proactive screening, identification and immunization of HIV-infected individuals. This program is based on a network of primary care/ public health providers and specialists working together to ensure the timely administration of recommended vaccines.

The Ligurian HIV Network web platform allows to identify the susceptible individuals, to monitor immunization 
di collegamento automatico tra la piattaforma web già esistente Ligurian HIV Network, che permette la gestione informatizzata dei pazienti, i singoli sistemi demografici ospedalieri e le anagrafi vaccinali informatizzate, con l'obiettivo di individuare i soggetti suscettibili, calcolare il numero di dosi somministrate e conoscere il denominatore per le coperture vaccinali. Come esempio di contestualizzazione territoriale del LIV in HIV, secondo un percorso multidisciplinare integrato, viene riportata l'esperienza maturata presso l'ambulatorio di Prevenzione delle Malattie Trasmissibili, Vaccinazioni e Sperimentazioni Cliniche dell'U.O. Igiene dell'Ospedale Policlinico San Martino- IST e gli ambulatori di Clinica di Malattie Infettive dell'Ospedale Policlinico San Martino- IST e dell'E.O. Ospedali Galliera, centri di riferimento per l'area metropolitana genovese. coverage and to manage failures and defaulting, using a connection with regional immunization registry system. The aim of this study is to describe the experience of the referral outpatient clinics of Infectious Disease (San Martino-IST hospital and Galliera hospital) and Hygiene Unit (San Martino-IST hospital) in Genoa metropolitan area, in order to evaluate the acceptability of vaccination in HIV-infected patients.

\section{La vaccinazione nel paziente con infe- zione da HIV}

L'introduzione delle attuali efficaci terapie antiretrovirali di combinazione (CART) ha progressivamente determinato un incremento dell'aspettativa di vita dei soggetti con infezione da virus dell'immunodeficienza umana (HIV) e migliorato le loro condizioni cliniche. Nella gestione di questi pazienti, il controllo delle malattie infettive prevenibili mediante vaccinazione rappresenta un aspetto cruciale (1).

Le raccomandazioni internazionali danno rilievo all'effettivo beneficio dell'immunoprofilassi nel prevenire infezioni, verso le quali questa categoria di pazienti presenta un rischio aumentato di complicanze; nel contempo deve essere valutata la peculiarità di questi soggetti, il cui deficit immunitario può determinare una risposta vaccinale subottimale ed una durata della protezione inferiore rispetto a quella della popolazione generale (2-4).

Nonostante vi siano per loro specifiche indicazioni e la disponibilità di preparati sicuri ed efficaci, i dati della letteratura segnalano coperture vaccinali non soddisfacenti sia a livello nazionale sia internazionale. In uno studio retrospettivo condotto da Tedaldi et al., solo il 32,4\% (198/612) e il $23,3 \%(167 / 716)$ dei pazienti candidabili alla vaccinazione anti-HBV ed anti-HAV, rispettivamente, avevano ricevuto almeno una dose di vaccino (5). I dati raccolti in Francia, su una coorte di 331 pazienti, hanno rilevato coperture inferiori rispetto a quelle della popolazione generale; le più frequenti motivazioni riportate sono state la mancata sensibilizzazione da parte del medico curante, la convinzione di non ottenere risultati in termini di efficacia ed il timore di reazioni avverse di tipo immuno-virologico (6). Anche da uno studio condotto a Londra su 75 bambini con infezione da HIV è emerso che questi sono a maggior rischio di incompleta immunizzazione rispetto ai coetanei sani (7). Analoghe valutazioni sono state fatte anche in Italia: un'indagine svolta in tre regioni (Lazio, Campania e Liguria), che aveva l'obiettivo di valutare i tassi di copertura vaccinale (CV) ed il ritardo nel completamento delle vaccinazioni obbligatorie e raccomandate in bambini portatori di alcune patologie croniche (diabete mellito di tipo 1, fibrosi cistica, disturbi neurologici, sindrome di Down ed infezione da HIV), ha rivelato che, su 275 pazienti, la CV a 24 mesi di vita era solo dell'85\% per Difterite-Tetano-Pertosse, Poliomielite ed Epatite B e del 62\% per Morbillo-Parotite e Rosolia (8). In particolare, I'analisi di sopravvivenza, corretta secondo il modello di Cox, ha evidenziato che, fra tutti i bambini inclusi nello studio, quelli con infezione da HIV hanno subito maggior ritardo nel completamento delle schedule vaccinali (8).

Le ragioni di questo fenomeno sono da ascriversi sia al fatto che questa popolazione rappresenta ancora oggi un target difficile da raggiungere sia perché l'approccio vaccinale in questi pazienti è reso incerto da molteplici dubbi, che gli operatori sanitari si pongono nella pratica clinica. Quelli più frequenti riguardano l'efficacia dei preparati 
vaccinali, la tempistica e la durata dell'immunità, il timore di effetti collaterali, la difficoltà nella valutazione del profilo del paziente e dei tempi di ripresa della funzionalità del sistema immunitario, I'insufficiente conoscenza delle attuali raccomandazioni, che indicano l'implementazione di interventi di immunizzazione per i gruppi a rischio (9-11).

Tutto questo comporta la collocazione della proposta vaccinale in secondo piano rispetto alla gestione della terapia antiretrovirale ed il trattamento delle co-morbosità.

Il profilo di efficacia dipende da differenti fattori, legati al preparato vaccinale, all'infezione da HIV e al grado di compromissione immunitaria dell'ospite (12). A questo riguardo, alcuni studi hanno correlato la risposta vaccinale alla conta dei linfociti T CD4+, alla carica virale e allo stadio della malattia (13-16). In generale, i dati sull'effectiveness delle vaccinazioni nella popolazione con infezione da HIV sono pochi e spesso si riferiscono a setting non confrontabili fra loro; gli studi di immunogenicità, condotti soprattutto in Paesi industrializzati, presentano numerosità limitata (17).

Analogamente, per quanto riguarda la durata della protezione, ci basiamo su lavori scientifici molto eterogenei fra di loro, in termini ad esempio di stato immunologico della popolazione in studio al momento dell'immunizzazione, di diversità degli antigeni vaccinali impiegati, di tempistica nella somministrazione delle terapie antiretrovirali rispetto alla vaccinazione. In una recente metanalisi, è stata sottoposta a revisione la letteratura relativa all'immunità a lungo termine in soggetti con infezione da HIV, che avevano precedentemente risposto al ciclo di immunizzazione primario, per le principali malattie prevenibili mediante vaccinazione: gli autori concludono che la durata della protezione in questi pazienti è più breve rispetto ai controlli, e che, soprattutto, una rilevante parte di questi ha perso il titolo protettivo prima che una dose booster venisse loro proposta (18). Tali considerazioni suggeriscono l'utilità di strategie atte ad implementare l'esecuzione di test sierologici, per individuare i soggetti suscettibili e programmare la somministrazione di eventuali dosi di richiamo o rivaccinazioni, anche se ulteriori dati a supporto saranno necessari in questo senso. La valutazione dell'opportunità di booster nell'età adulta, per le vaccinazioni ricevute durante l'infanzia e l'adolescenza, può rappresentare ulteriore spunto per la ricerca futura (19).

Relativamente alla tempistica, ci si chiede quale sia il momento migliore per vaccinare, dubbio riferito in particolare ai soggetti che devono iniziare la terapia antiretrovirale. Gli studi condotti al riguardo sono pochi e peraltro poco consistenti (17). Supposto che l'immuno-ricostituzione indotta dalla terapia aumenti l'efficacia del preparato vaccinale somministrato (20), la scelta di attendere una conta adeguata di linfociti T CD4 e una bassa viremia sembrerebbe quella più opportuna; d'altro canto, ogni vaccinazione procrastinata rappresenta una mancata opportunità di immunizzazione, per cui ci si assume il rischio di una potenziale esposizione o di perdita del paziente al follow-up $(21,22)$.

Un altro territorio di incertezza è quello relativo alla sicurezza dei preparati vaccinali. Sulla base dei dati pubblicati in letteratura, il profilo di sicurezza dei vaccini inattivati nei soggetti con infezione da HIV sembra essere sovrapponibile a quello della popolazione sana (22). Benché non siano stati condotti studi con una potenza sufficiente per rilevare la comparsa di eventi avversi rari, i dati post-marketing non suggeriscono di porre particolare attenzione a questa categoria di soggetti (22). Quando disponibili, i vaccini inattivati devono essere preferiti ai vivi ed attenuati. Questi ultimi sono controindicati solo in caso di pazienti con bassa conta dei linfociti T CD4+; fa eccezione la vaccinazione con bacillo di Calmette e Guérin, per la quale non abbiamo sufficienti dati di sicurezza. Anche la preoccupazione che lo stimolo vaccinale possa favorire la replicazione virale rappresenta talvolta motivo di esitazione da parte degli operatori sanitari.

Questo fenomeno, osservato dopo la vaccinazione anti-influenzale, è transitorio e un ampio studio, su oltre 30.000 soggetti immunizzati, non ha mostrato esiti negativi a lungo termine sulla conta dei linfociti T CD4, sull'HIV RNA plasmatico e sulla progressione della malattia $(22,23)$. Per questa ragione, i benefici ottenuti con la vaccinazione continuano a superare i rischi teorici esistenti.

La vaccinazione rappresenta un'eccellente opportunità di prevenzione ed il raggiungimento di coperture vaccinali adeguate in questi pazienti è 
una sfida che deve essere affrontata attraverso la predisposizione di piani operativi per promuovere l'offerta attiva delle vaccinazioni prioritarie e l'attivazione di strategie selettive, per l'identificazione ed il raggiungimento dei soggetti suscettibili.
Con questa finalità, le attuali raccomandazioni internazionali, sia negli Stati Uniti, tra cui ad esempio quelle dei Centers of Diseases Control and Prevention (CDC) (24), dell'Advisory Committee on Immunization Practices (ACIP) (4), dell'HIV Medicine

Tabella 1. Raccomandazioni delle principali società scientifiche per le vaccinazioni in soggetti adulti con infezione da HIV, con vaccini inattivati (adattato da Crum-Cianflone et al. 19).

\section{VACCINI INATTIVATI 1. Indicazione, 2. Tempistica e modalità di somministrazione}

\begin{tabular}{|c|c|c|c|c|}
\hline Vaccino & Europa $^{26}$ & Stati Uniti ${ }^{3,4,24,25}$ & Regno Unito² & Italia ${ }^{27}$ \\
\hline Influenza & $\begin{array}{l}\text { 1. Tutti } \\
\text { 2. Annuale }\end{array}$ & $\begin{array}{l}\text { 1. Tutti } \\
\text { 2. Annuale }\end{array}$ & $\begin{array}{l}\text { 1. Tutti } \\
\text { 2. Annuale }\end{array}$ & $\begin{array}{l}\text { 1. Tutti } \\
\text { 2. Annuale }\end{array}$ \\
\hline Pneumococco & $\begin{array}{l}\text { 1. Tutti } \\
\text { 2. Una dose di PCV } 13\end{array}$ & $\begin{array}{l}\text { 1. Tutti } \\
\text { 2. Una dose di PCV13 seguita } \\
\text { da una dose di PPV23 dopo } \\
\text { almeno } 8 \text { settimane. } \\
\text { Ripetere una dose di } \\
\text { PPV23 } 5 \text { anni dopo; se già } \\
\text { vaccinato con PPV23, som- } \\
\text { ministrare PCV13 a distanza } \\
\text { di almeno un anno, seguito } \\
\text { da una dose di PPV23 dopo } \\
\text { almeno } 5 \text { anni }\end{array}$ & $\begin{array}{l}\text { 1. Tutti } \\
\text { PPV23 raccomandato in } \\
\text { soggetti con ulteriori fattori } \\
\text { di rischio che permangono } \\
\text { nel tempo (es. asplenia), } \\
\text { sulla base delle raccomanda- } \\
\text { zioni previste dai programmi } \\
\text { nazionali } \\
\text { 2. Una dose di PCV-13. PPV23 } \\
\text { somministrato almeno } 3 \\
\text { mesi dopo PCV13 }\end{array}$ & $\begin{array}{l}\text { 1. Tutti } \\
\text { 2. Almeno una dose di PCV13 } \\
\text { seguita da una dose di } \\
\text { PPV23 dopo almeno } 8 \\
\text { settimane; se già vaccinato } \\
\text { in precedenza con PPV23, } \\
\text { somministrare PCV13 a } \\
\text { distanza di un anno. } \\
\text { Considerare una dose di } \\
\text { PPV23 dopo almeno } 5 \text { anni } \\
\text { dalla precedente dose di } \\
\text { PPV23 }\end{array}$ \\
\hline Epatite $B$ (HBV) & $\begin{array}{l}\text { 1. Tutti i soggetti non immuni } \\
\text { 2. Nei soggetti non-respon- } \\
\text { ders (anti-HBs<10 mlU/ } \\
\text { ml dopo il ciclo primario): } \\
4 \text { dosi a } 0,1,6,12 \text { mesi. } \\
\text { (dose da } 40 \mathrm{mcg} \text { ) } \\
\text { Non vengono fornite } \\
\text { ulteriori specifiche }\end{array}$ & $\begin{array}{l}\text { 1. Tutti i soggetti non immuni } \\
\text { 2. Ciclo primario a } 3 \text { dosi: } \\
0,1,6 \text { mesi. ( } 20 \mathrm{mcg}) \text { o } 4 \\
\text { dosi: } 0,1,2,6 \text { mesi ( } 40 \mathrm{mcg} \text { ) }\end{array}$ & $\begin{array}{l}\text { 1. Tutti i soggetti non immuni. } \\
\text { 2. Ciclo primario a } 4 \text { dosi (40 } \\
\text { mcg): } 0,1,2,6 \text { mesi. Nei } \\
\text { soggetti con CD4>500 } \\
\text { cells/mm }{ }^{3} \text { considerare ciclo } \\
\text { ultrarapido in } 3 \text { settimane. } \\
\text { (3 dosi standard a } 20 \mathrm{mcg} \text { ) } \\
\text { Raccomandata la sommi- } \\
\text { nistrazione di richiami se il } \\
\text { titolo anticorpale scende al } \\
\text { di sotto di } 10 \text { IU/L }\end{array}$ & $\begin{array}{l}\text { 1. Tutti i soggetti non immuni } \\
\text { 2. Schema a tre o quattro dosi. } \\
\text { Misurare il titolo anticorpale } \\
\text { a } 4-8 \text { settimane dal termine } \\
\text { del ciclo basale e successi- } \\
\text { vamente ogni 1-2 anni. }\end{array}$ \\
\hline & & & & \\
\hline
\end{tabular}




\section{VACCINI INATTIVATI 1. Indicazione, 2. Tempistica e modalità di somministrazione}

\begin{tabular}{|c|c|c|c|c|}
\hline $\begin{array}{c}\text { Difterite, tetano, } \\
\text { pertosse (Dtp) }\end{array}$ & Nessuna raccomandazione & $\begin{array}{l}\text { 1. Tutti } \\
\text { 2. Dose Td booster ogni } 10 \\
\text { anni. La vaccinazione con } \\
\text { Tdap è raccomandata in } \\
\text { caso di: } \\
\text { - Gravidanza ( } 27-39 \text { setti- } \\
\text { mane): una dose di Tdap a } \\
\text { ogni gravidanza } \\
\text { - Contatto stretto previsto } \\
\text { con bambino < } 12 \text { mesi: } 1 \\
\text { dose di Tdap }\end{array}$ & $\begin{array}{l}\text { 1. Tutti } \\
\text { 2. Td booster ogni } 10 \text { anni, } \\
\text { ogni } 5 \text { anni nei soggetti con } \\
\text { età }>50 \text { anni. } \\
\text { Fattori di rischio per } \\
\text { pertosse: } \\
\text { - Gravidanza ( } 28 \text { - } 32 \\
\text { settimane): una dose di } \\
\text { Tdap/IPV } \\
\text { - In caso di epidemia }\end{array}$ & $\begin{array}{l}\text { 1. Stesse indicazioni della } \\
\text { popolazione adulta HIV } \\
\text { negativa } \\
\text { 2. Tdap booster ogni } 10 \text { anni. } \\
\text { Vaccinazione con Tdap nel- } \\
\text { le gravide ( } 28^{\circ} \text { settimana) }\end{array}$ \\
\hline Epatite A (HAV) & $\begin{array}{l}\text { 1. Soggetti non immuni con } \\
\text { condizioni di rischio: } \\
\text { - Viaggi in aree endemiche } \\
\text { - MSM } \\
\text { - Uso di sostanze per via } \\
\text { iniettiva } \\
\text { - Infezioni da HBV e/o HCV } \\
\text { 2. Due dosi. Nessuna indica- } \\
\text { zione per dosi booster }\end{array}$ & $\begin{array}{l}\text { 1. Soggetti non immuni con } \\
\text { condizioni di rischio: } \\
\text { - Malattia epatica cronica } \\
\text { - Infezioni da HBV e/o HCV } \\
\text { - Uso di sostanze per via } \\
\text { iniettiva e non } \\
\text { - MSM } \\
\text { - Rischio occupazionale } \\
\text { - Viaggi in aree endemiche } \\
\text { 2. Due dosi. Controllo } \\
\text { anticorpi IgG a } 1 \text { mese dal } \\
\text { ciclo primario. Nei soggetti } \\
\text { no-responders: booster se } \\
\text { conta CD4>200 cells/mm } 3 \mathrm{~mm}^{3}\end{array}$ & $\begin{array}{l}\text { 1. Soggetti non immuni con } \\
\text { condizioni di rischio: } \\
\text { - Conviventi e partner di } \\
\text { persone infette } \\
\text { - Viaggi in aree endemiche } \\
\text { - MSM } \\
\text { - Uso di sostanze per via } \\
\text { iniettiva e non } \\
\text { - Rischio di epidemia } \\
\text { - Rischio occupazionale } \\
\text { - Emofilia } \\
\text { - Lungodegenti e personale } \\
\text { di assistenza } \\
\text { 2. Soggetti con conta CD4>350 } \\
\text { cells/mm³: due dosi a } 0 \text { e } \\
6 \text { mesi. Soggetti con conta } \\
\text { CD4<350 cells/mm³: tre dosi } \\
\text { a 0,1,6 mesi. Dose booster } \\
\text { ogni } 10 \text { anni se permangono } \\
\text { condizioni di rischio }\end{array}$ & $\begin{array}{l}\text { 1. Soggetti non immuni con } \\
\text { condizioni di rischio: } \\
\text { - Viaggi in aree endemiche } \\
\text { - MSM } \\
\text { - Uso di sostanze per via } \\
\text { iniettiva } \\
\text { - Epatopatie croniche } \\
\text { - Emofilie } \\
\text { - Infezioni da HBV e/o HCV } \\
\text { 2. Due dosi. Rivaccinare i } \\
\text { no-responders. } \\
\text { Considerare l'uso del } \\
\text { vaccino combinato per HBV } \\
\text { e HAV }\end{array}$ \\
\hline $\begin{array}{c}\text { Papillomavirus } \\
\text { (HPV) }\end{array}$ & Nessuna raccomandazione & $\begin{array}{l}\text { 1. Tutti i soggetti maschi e } \\
\text { femmine di età compresa } \\
\text { tra } 9 \text { e } 26 \text { anni. } \\
\text { 2. Tre dosi a } 0,1-2,6 \text { mesi con } \\
\text { 4-HPV o 9-HPV }\end{array}$ & $\begin{array}{l}\text { 1. Tutti i soggetti: } \\
\text { - Maschi di età compresa } \\
\text { tra } 9 \text { e } 26 \text { anni } \\
\text { - MSM di età< } 40 \text { anni } \\
\text { - Femmine di età< } 40 \text { anni } \\
\text { - Storia di lesioni da HPV di } \\
\text { alto grado } \\
\text { 2. Tre dosi a 0, 1-2, } 6 \text { mesi } \\
\text { preferibilmente con 9-HPV }\end{array}$ & $\begin{array}{l}\text { 1. Tutti i soggetti a partire } \\
\text { dai } 9 \text { anni di età secondo } \\
\text { le indicazioni delle schede } \\
\text { tecniche. } \\
\text { 2. Tre dosi con 4-HPV o 9-HPV }\end{array}$ \\
\hline
\end{tabular}

(CONTINUA) 


\section{VACCINI INATTIVATI 1. Indicazione, 2. Tempistica e modalità di somministrazione}

\begin{tabular}{|c|c|c|c|c|}
\hline $\begin{array}{c}\text { Meningococco } \\
\text { ACWY } \\
\text { (MenACWY) }\end{array}$ & $\begin{array}{l}\text { 1. Vaccinazione secondo } \\
\text { le raccomandazioni } \\
\text { valide per la popolazione } \\
\text { generale } \\
\text { 2. Due dosi a distanza di 1-2 } \\
\text { mesi. Dose booster ogni } \\
5 \text { anni }\end{array}$ & $\begin{array}{l}\text { 1. Tutti } \\
\text { 2. Due dosi a distanza di } \\
\text { almeno mesi. Dose booster } \\
\text { ogni } 5 \text { anni }\end{array}$ & $\begin{array}{l}\text { 1. Tutti i soggetti con i seguenti } \\
\text { fattori di rischio: } \\
\text { - Età < } 25 \text { anni e non prece- } \\
\text { dentemente vaccinati, con } \\
\text { anamnesi vaccinale incerta } \\
\text { o che abbiano ricevuto } \\
\text { I'ultima dose di vaccino } \\
\text { MenC a età < } 10 \text { anni } \\
\text { (Men ACWY e MenB) } \\
\text { - Asplenia o deficit del } \\
\text { complemento } \\
\text { - Viaggi in aree endemiche } \\
\text { - Esposizione durante } \\
\text { un'epidemia } \\
\text { 2. Due dosi a distanza di } 2 \\
\text { mesi. booster ogni } 5 \text { anni } \\
\text { se permangono condizioni } \\
\text { di rischio }\end{array}$ & $\begin{array}{l}\text { 1. Tutti i soggetti con i seguenti } \\
\text { fattori di rischio: } \\
\text { - Asplenia o deficit } \\
\text { splenico } \\
\text { - Deficit di fattori del } \\
\text { complemento } \\
\text { - Trattamento con Eculizumab } \\
\text { - MSM } \\
\text { - Viaggi in aree endemiche } \\
\text { - Rischio professionale } \\
\text { - Età compresa tra } 18 \text { e } \\
25 \text { anni } \\
\text { 2. Due dosi a distanza di 8-12 } \\
\text { settimane }\end{array}$ \\
\hline $\begin{array}{c}\text { Meningococco } \\
\text { B (MenB) }\end{array}$ & $\begin{array}{l}\text { 1. Vaccinazione secondo } \\
\text { le raccomandazioni } \\
\text { valide per la popolazione } \\
\text { generale }\end{array}$ & $\begin{array}{l}\text { 1. Nessuna raccomandazione } \\
\text { specifica per persone senza } \\
\text { ulteriori fattori di rischio }\end{array}$ & $\begin{array}{l}\text { 1. Tutti i soggetti con i seguenti } \\
\text { fattori di rischio: } \\
\text { - Età <25 anni non prece- } \\
\text { dentemente vaccinati, con } \\
\text { anamnesi vaccinale incerta } \\
\text { o che abbiano ricevuto } \\
\text { ultima dose di vaccino } \\
\text { MenC a età<10 anni } \\
\text { - Asplenia o deficit del } \\
\text { complemento } \\
\text { - Esposizione durante } \\
\text { un'epidemia }\end{array}$ & $\begin{array}{l}\text { 1. Tutti i soggetti con i seguenti } \\
\text { fattori di rischio: } \\
\text { - Asplenia o deficit } \\
\text { splenico } \\
\text { - Deficit di fattori del } \\
\text { complemento } \\
\text { - Trattamento con Eculizumab } \\
\text { - MSM } \\
\text { - Viaggi in aree endemiche } \\
\text { - Rischio professionale } \\
\text { - Età compresa tra } 18 \text { e } \\
25 \text { anni } \\
\text { 2. Due dosi a distanza di } \\
\text { almeno un mese. Non } \\
\text { previste dosi booster }\end{array}$ \\
\hline
\end{tabular}

Association of the Infectious Diseases Society of America (25) e dell'Infectious Diseases Society of America (IDSA) (3), sia in Europa del Paediatric European Network for Treatment of AIDS (PENTA) (17), dell'European AIDS Clinical Society (EACS) (26) e della British HIV Association (BHIVA) (2), indicano le vaccinazioni prioritarie nei soggetti con infezione da HIV, specificando operativamente do- saggio, numero di dosi, tempistiche e modalità di somministrazione.

Suddivise per nazionalità di provenienza, sono riportate le raccomandazioni delle principali società scientifiche nazionali (27) ed internazionali $(2,3,4,24-26)$ per vaccini inattivati (Tabella 1 ), vivi e attenuati (Tabella 2) per la popolazione adulta con infezione da HIV (19). 


\section{VACCINI VIVI ATTENUATI 1. Indicazione, 2. Tempistica e modalità di somministrazione}

\begin{tabular}{|c|c|c|c|c|}
\hline Vaccino & Europa $^{26}$ & Stati Uniti ${ }^{3,4,24,25}$ & Regno Unito ${ }^{2}$ & Italia ${ }^{27}$ \\
\hline $\begin{array}{c}\text { Varicella } \\
\text { (VZV) }\end{array}$ & $\begin{array}{l}\text { 1. Tutti i soggetti VZV } \\
\text { sieronegativi e con conta } \\
\text { dei CD4>200 cells } / \mathrm{mm}^{3}\end{array}$ & $\begin{array}{l}\text { 1. Tutti i soggetti VZV } \\
\text { sieronegativi e con conta } \\
\text { dei CD4>200 cells } / \mathrm{mm}^{3} \\
\text { 2. Due dosi a distanza di } 3 \\
\text { mesi }\end{array}$ & $\begin{array}{l}\text { 1. Tutti i soggetti VZV } \\
\text { sieronegativi e con conta } \\
\text { dei CD4 }>200 \text { cells } / \mathrm{mm}^{3} \mathrm{e} \\
\text { preferibilmente in ART } \\
\text { 2. Due dosi a distanza di } 3 \\
\text { mesi. Controllo titolo anticor- } \\
\text { pale a } 4-6 \text { settimane dalla } \\
\text { seconda dose } \\
\text { Post-esposizione: se sierone- } \\
\text { gativo e conta dei CD4>200 } \\
\text { cells/mm³: una dose entro } \\
3-5 \text { giorni dall'esposizione e } \\
\text { seconda dose a } 3 \text { mesi }\end{array}$ & $\begin{array}{l}\text { 1. Tutti i soggetti VZV sierone- } \\
\text { gativi e con conta dei CD4 } \\
>200 \text { cells } / \mathrm{mm}^{3} \text { e preferibil- } \\
\text { mente }>400 \text { cells } / \mathrm{mm}^{3} \\
\text { 2. Due dosi a distanza di } \\
\text { almeno } 6-8 \text { settimane }\end{array}$ \\
\hline $\begin{array}{c}\text { Morbillo } \\
\text {-parotite } \\
\text {-rosolia (MPR) }\end{array}$ & $\begin{array}{l}\text { 1. Controindicato in caso di } \\
\text { conta dei CD } 4<200 \text { cells/ } \\
\mathrm{mm}^{3} \text { e/o AIDS }\end{array}$ & $\begin{array}{l}\text { 1. Solo soggetti con conta } \\
\text { dei CD4>200 cells } / \mathrm{mm}^{3} \text { da } \\
\text { almeno } 6 \text { mesi } \\
\text { 2. Due dosi a distanza di } \\
\text { almeno } 28 \text { giorni }\end{array}$ & $\begin{array}{l}\text { 1. Tutti i soggetti sieronegati- } \\
\text { vi e con conta dei CD4>200 } \\
\text { cells/mm³ e clinicamente } \\
\text { stabili } \\
\text { 2. Due dosi a distanza di } \\
\text { almeno un mese } \\
\text { In caso di esposizione a } \\
\text { morbillo e con conta dei } \\
\text { CD4>200 cells/mm }{ }^{3} \text { in } \\
\text { soggetti sieronegativi } \\
\text { preferibilmente in terapia } \\
\text { ART o soppressione virale: } \\
\text { una dose entro } 3 \text { giorni } \\
\text { da esposizione. Donne in } \\
\text { età fertile sieronegative } \\
\text { per rosolia e con conta dei } \\
\text { CD4>200 cells/mm³ e non } \\
\text { in gravidanza: una dose } \\
\text { seguita da controllo titolo } \\
\text { anticorpale dopo } 4 \text { settima- } \\
\text { ne, o } 2 \text { dosi a distanza di } \\
\text { almeno un mese }\end{array}$ & $\begin{array}{l}\text { 1. Tutti i soggetti sieronega- } \\
\text { tivi stabili clinicamente } \\
\text { e con conta dei CD4>200 } \\
\text { cells } / \mathrm{mm}^{3} \\
\text { 2. Due dosi a distanza di } \\
\text { almeno un mese }\end{array}$ \\
\hline Herpes Zoster (HZ) & Nessuna raccomandazione & 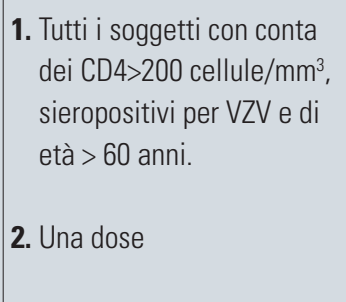 & $\begin{array}{l}\text { 1. Tutti i soggetti con conta } \\
\text { dei CD4 }>200 \text { cells } / \mathrm{mm}^{3} \text {, } \\
\text { di età }>70 \text { anni, sieropositivi } \\
\text { per VZV, preferibilmente in } \\
\text { terapia ART } \\
\text { 2. Una dose }\end{array}$ & Nessuna raccomandazione \\
\hline
\end{tabular}

Tabella 2. Raccomandazioni delle principali società scientifiche per le vaccinazioni in soggetti adulti con infezione da HIV, con vaccini vivi ed attenuati. (adattato da Crum-Cianflone et al. 19). 


\section{Il Ligurian Vaccination in HIV Program: un percorso dedicato e condiviso}

La Regione Liguria, sempre attenta al controllo delle malattie prevenibili mediante immunizzazione ed in linea con gli obiettivi del Piano Nazionale in Prevenzione Vaccinale (PNPV) 2017-19, che prevedono, fra gli altri, la garanzia dell'offerta attiva e gratuita delle vaccinazioni nei soggetti a rischio e la promozione di iniziative atte a implementare le vaccinazioni nei gruppi difficili da raggiungere (28), ha predisposto un calendario vaccinale rivolto alla popolazione con infezione da HIV, ad integrazione del Piano Regionale in Prevenzione Vaccinale (PRPV) (29) (Delibera di Giunta Regionale n.1314 del 30/11/2015) (30). L'offerta è in larga parte sovrapponibile a quella prevista per la popolazione generale. Le principali differenze riguardano la vaccinazione antimeningococcica sia con preparato quadrivalente coniugato (Men ACWY), che si preferisce al monovalente $\mathrm{C}$, sia contro il Meningococco di gruppo $B$, nei bambini e negli adulti. Ė inoltre previsto l'ampliamento dell'offerta attiva e gratuita della vaccinazione contro il Papillomavirus (HPV) con formulazione quadrivalente, inserendo la gratuità a tutte le età (compatibilmente con la scheda tecnica), oltre alle coorti già previste dal PRPV. Non sono disponibili dati in donne di età superiore ai 45 anni ed in uomini di età superiore ai 26 anni.

Tuttavia la vaccinazione può essere presa in considerazione anche nei maschi fino ai 45 anni (31). Tali indicazioni dovranno adeguarsi alla recente disponibilità del preparato nove-valente. Nella Tabella 3 si riportano le indicazioni in soggetti con infezione da HIV per l'infanzia e per l'adolescenza (0-17 anni) e nella Tabella 4 per gli adulti e per gli anziani.

La predisposizione di un apposito calendario e l'offerta in gratuità sono indispensabili per l'implementazione delle attività vaccinali, ma non sufficienti per migliorare la compliance in queste persone che, ancora oggi per la paura di essere stigmatizzate, evitano di rivolgersi ai servizi vaccinali e di dichiarare la propria condizione. Questa è una delle problematiche che rende tale popolazione tanto difficile da raggiungere. È pertanto necessario che l'approccio vaccinale sia coordinato e multidisciplinare, che i servizi lavorino in maniera coerente tra di loro e in sinergia con altre professionalità.
Il Llgurian Vaccination in HIV Program (LIV in HIV) è un'iniziativa su base regionale per la ricerca attiva, l'individuazione ed il raggiungimento dei soggetti suscettibili per le malattie prevenibili mediante vaccinazione. È stato creato un percorso assistenziale condiviso, che ha coinvolto gli operatori dei centri vaccinali, dei centri di riferimento per la diagnosi e cura di questi pazienti, dei pediatri di libera scelta e dei medici di medicina generale, nell'ambito di una collaborazione strutturata a livello regionale.

La presentazione del progetto è avvenuta in tre edizioni, separatamente nelle tre macro-aree liguri del Centro, Levante e Ponente (Genova, La Spezia e Savona) per favorire, mediante la contestualizzazione territoriale e comunque secondo un unico percorso di condivisione regionale, l'attivazione di canali di comunicazione ed incontri sistematici tra le figure professionali coinvolte nella retention in care del paziente e gli operatori dei servizi vaccinali.

Per il monitoraggio dell'andamento del programma, è fondamentale dare una dimensione alla popolazione target (così difficile da identificare nell'ambito di quella generale), ed è anche necessario conoscere la proporzione delle persone attivamente invitate alla vaccinazione e la proporzione di coloro che la rifiutano, avere una stima dei soggetti suscettibili, calcolare le coperture vaccinali ed essere in grado di riscontrare eventuali fallimenti vaccinali.

La piattaforma web Ligurian HIV Network, sviluppata nel 2013 su un modello di architettura software orientata ai servizi (service-oriented-architecture-SOA), permette la gestione informatizzata dei pazienti che partecipano a studi multicentrici, tramite registrazione degli aggiornamenti sul loro stadio clinico ed acquisizione degli esami di laboratorio. Questo tipo di architettura prevede I'alimentazione dei dati sia automaticamente, da parte dei sistemi informatici di alcuni ospedali (ad esempio, referti di indagini di laboratorio effettuate) sia in maniera manuale, direttamente da parte degli operatori che accedono al sistema (32).

Le informazioni dai vari centri vengono convogliate in un unico archivio condiviso. L'interfaccia del sistema si articola in una parte pubblica, con contenuti divulgativi sul progetto, ed una parte privata, attraverso la quale gli operatori possono direttamente consultare la cartella clinica del paziente, 


\section{articolo originale}

\begin{tabular}{|c|c|c|c|c|c|c|c|c|c|c|c|c|}
\hline Vaccino & Nascita & $\begin{array}{l}3^{\circ} \text { mese } \\
\left(61^{\circ} \text { giorno) }\right.\end{array}$ & $\begin{array}{l}3^{\circ} \text { mese } \\
\left(76^{\circ} \text { giorno) }\right.\end{array}$ & $\begin{array}{c}4^{\circ} \text { mese } \\
\text { (106 giorno) }\end{array}$ & $\begin{array}{r}\mathbf{5}^{\circ} \mathbf{m e s e} \\
\text { ) }\left(121^{\circ} \text { giorno }\right.\end{array}$ & $\begin{array}{c}\mathbf{6}^{\circ} \text { mese } \\
\left(151^{\circ} \text { giorno }\right)\end{array}$ & $12^{\circ}$ mese & $13^{\circ}$ mese & $15^{\circ} \mathrm{mese}$ & $\begin{array}{c}16^{\circ} \text { mese } \\
-4 \text { anni }\end{array}$ & $\begin{array}{c}5-6 \\
\text { anni }\end{array}$ & $\begin{array}{l}11-17 \\
\text { anni }\end{array}$ \\
\hline DTPa & & $\mathrm{DTPa}$ & & & $\mathrm{DTPa}$ & & $\mathrm{DTPa}$ & & & & $\mathrm{DTPa}$ & dTpa \\
\hline IPV & & IPV & & & IPV & & IPV & & & & IPV & IPV \\
\hline$H B V$ & $\mathrm{HBV}^{1}$ & HBV & & & HBV & & HBV & & & & & \\
\hline Hib & & Hib & & & $\mathrm{Hib}$ & & $\mathrm{Hib}$ & & & & & \\
\hline Pneumococco & & PCV13 & & & PCV13 & & PCV13 & & & & \multicolumn{2}{|c|}{ PCV13/PPV23 } \\
\hline Men B & & & Men B & Men B & & Men B & & & Men B & & & \\
\hline Men ACWY & & & & & & & & $\begin{array}{c}\text { Men } \\
\text { ACWY } \\
\text { (coniugato) }^{4}\end{array}$ & & & & 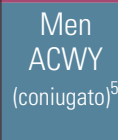 \\
\hline MPR & & & & & & & & $\mathrm{MPR}^{2}$ & & & \multirow{2}{*}{$\begin{array}{c}\text { MPR2 }^{2} \\
+\operatorname{Var}^{6} 0 \\
\text { MPRV } \\
2,6\end{array}$} & \multirow{2}{*}{$\begin{array}{c}\text { MPR }^{2} \\
+\quad+r^{6,7} \\
\text { o MPRV } \\
2,6,7\end{array}$} \\
\hline Varicella & & & & & & & & & $\operatorname{Var}^{6}$ & & & \\
\hline HPV & & & & & & & & & & & & $\mathrm{HPV} 4^{8}$ \\
\hline HAV & & & & & & & & \multicolumn{5}{|c|}{ HAV-2 dosi (0, 6-12 mesi) } \\
\hline Influenza & & & & & & & nfluenza vac & accinazione & annuale (a & partire dai 6 & mesi di v & \\
\hline
\end{tabular}

Tabella 3. Calendario vaccinale ligure per l'infanzia e per l'adolescenza, in soggetti con infezione da HIV (0-17 anni).

${ }^{1}$ In bimbi nati da madre HBSAg positive: somministrazione entro le prime 12-24 ore di vita, contemporaneamente alle immunoglobuline specifiche antiepatite B, la prima dose di vaccino anti-HBV, il ciclo andrà completato con una seconda dose a distanza di 4 settimane dalla prima, con una terza dose dopo il compimento della ottava settimana e con la quarta dose in un periodo compreso tra l'undicesimo ed il dodicesimo mese di vita.

${ }^{2}$ La vaccinazione antimorbillo-rosolia-parotite è raccomandata per bambini con infezione da HIV asintomatica o lievemente sintomatica, ma è controindicata per persone con severa immunodepressione da HIV o da qualsiasi altro disordine immunodepressivo.

${ }^{3}$ In riferimento ai focolai epidemici in corso, si ritiene opportuno, oltre al recupero dei soggetti suscettibili in questa fascia di età (catch up) anche una ricerca attiva ed immunizzazione dei soggetti conviventi/contatto, non vaccinati (mop up).

${ }^{4}$ Si raccomanda l'utilizzo del prodotto indicato per questa fascia di età.

${ }^{5}$ Dose singola in offerta attiva e gratuita alla coorte 14-15 anni, compresi i soggetti vaccinati nell'infanzia quando raggiungeranno tale eta'. Rimane la possibilità di somministrare il vaccino monovalente coniugato antimeningococco $\mathrm{C}$ per coloro che non volessero aderire all'offerta del quadrivalente.

${ }^{6}$ La vaccinazione anti-varicella è raccomandata per bambini con infezione da HIV asintomatica o lievemente sintomatica, ma è controindicata per persone con severa immunodepressione da HIV o da qualsiasi altro disordine immunodepressivo.

${ }^{7}$ Due dosi di vaccino MPRV per i soggetti mai vaccinati in precedenza. Per i soggetti vaccinati con 2 dosi di MPR, somministrare 2 dosi di vaccino antivaricella, a distanza di un mese I'una dall'altra. Per i soggetti che hanno già contratto la varicella, somministrare 2 dosi di vaccino MPR. Per i soggetti che hanno ricevuto 1 dose di vaccino MPR e 1 dose di vaccino anti-varicella, somministrare 1 dose di MPRV. Per i soggetti che hanno ricevuto 2 dosi di vaccino MPR e 1 dose di vaccino anti-varicella, somministrare 1 dose di vaccino anti-varicella.

${ }^{8}$ L'offerta riguarda tutti i bambini con infezione da HIV, maschi e femmine, nel dodicesimo anno di vita secondo RCP del vaccino in uso.

${ }^{9} 2$ dosi ripetute a distanza di almeno 4 settimane per bambini che vengono vaccinati per la prima volta, in formulazione pediatrica o mezza dose adulti (0,25 ml) fino a 36 mesi, a dose intera (0,5 ml) dopo i tre anni di vita.

\section{Legenda:}

DTPa: vaccino antidifto-tetanico-pertossico acellulare

dTpa: vaccino antidifto-tetanico-pertossico acellulare per adolescenti ed adulti

IPV: vaccino antipolio inattivato

HBV: vaccino antiepatite $B$

Hib: vaccino contro le infezioni invasive da Haemophilus influenzae tipo b

MPR: vaccino antimorbillo-parotite-rosolia

PCV13: vaccino antipneumococcico coniugato 13-valente

Men C: vaccino antimeningococco $\mathrm{C}$ coniugato

Men ACWY: vaccino antimeningococco A, C, W135, Y coniugato

Men B: meningococco B

HPV: vaccino antipapilloma virus

HAV: vaccino antiepatite A

Offerta attiva e gratuita

Raccomandato in presenza di fattori di rischio

(clinico, epidemiologico, viaggiatori internazionali, stile di vita o altro)

Recupero dei soggetti suscettibili in questa fascia di età (catch up) 


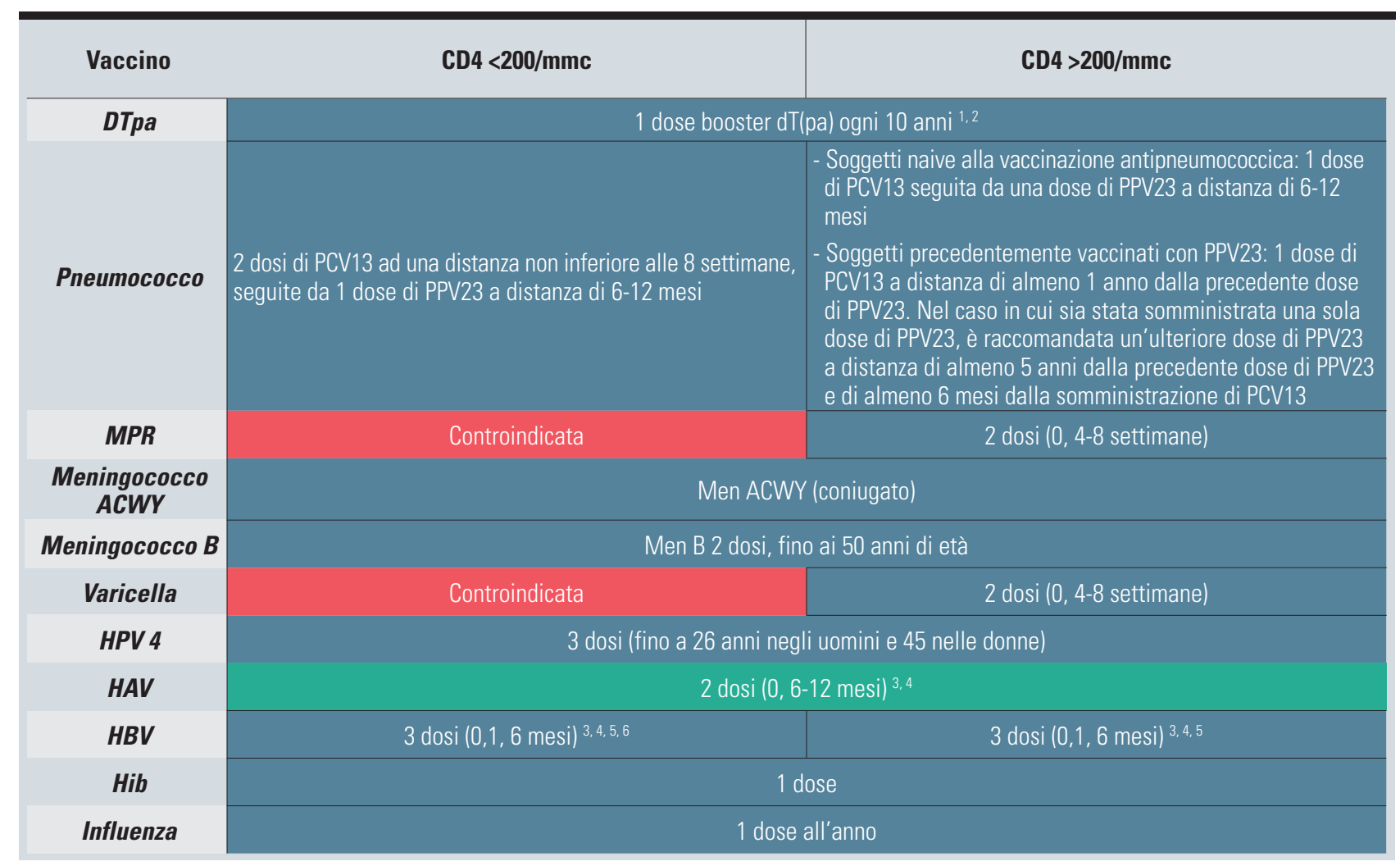

Tabella 4. Calendario vaccinale ligure per gli adulti e per gli anziani, in soggetti con infezione da HIV

'Si raccomanda test sierologico per il tetano ogni 5 anni, per valutare eventuale necessità di anticipare dose booster.

${ }^{2}$ Gli adulti con anamnesi incerta per il ciclo primario di vaccinazione con dT devono iniziare o completare la vaccinazione primaria. Un ciclo primario per adulti e composto da 2 dosi di vaccino contenente tetano e difterite (dT) e una terza dose con vaccino dTpa. Le prime 2 dosi devono essere somministrate a distanza di almeno 4 settimane l'una dall'altra e la terza dose 6-12 mesi dopo la seconda. I successivi richiami devono essere effettuati ogni 10 anni (a partire dal completamento della serie primaria) e almeno una delle dosi booster di vaccino dT dovrebbe essere rimpiazzata da 1 dose di vaccino dTpa.

${ }^{3}$ Dopo screening sierologico prevaccinale.

${ }^{4}$ Si ricorda la possibilità di utilizzare il vaccino con formulazione bivalente, anti-epatite A ed anti-epatite $B$.

${ }_{5}^{5}$ Si raccomanda valutazione sierologica al termine del ciclo vaccinale, per documentare adeguata sieroconversione. In particolare, in caso di titolo anti-HBs: $<10 \mathrm{~m} / \mathrm{U} / \mathrm{mL}$, ripetere ciclo vaccinale completo e successiva rivalutazione sierologica.

10-100 m/U/mL, una dose booster se il paziente è immunocompetente, in caso di immunocompromissione procrastinare a 6 mesi dal recupero immunologico.

$>100 \mathrm{mIJ} / \mathrm{mL}$, protezione a lungo termine. E' comunque raccomandata rivalutazione sierologico ogni 5 anni, annualmente in caso di immunocompromissione.

${ }^{6}$ valutare I'utilizzo dose vaccino anti-epatite $B$ doppia.

\section{Legenda:}

DTpa: vaccino antidifto-tetanico-pertossico acellulare per adolescenti ed adulti

HBV: vaccino antiepatite B

MPR: vaccino antimorbillo-parotite-rosolia

PCV13: vaccino antipneumococcico coniugato 13-valente

Men ACWY: vaccino antimeningococco A, C, W135, Y

HPV: vaccino antipapilloma virus

HAV: vaccino antiepatite A

Hib: vaccino contro le infezioni invasive da Haemophilus influenzae b

Men B : meningococco B

Offerta attiva e gratuita

Vaccinazione controindicata

Raccomandato in presenza di fattori di rischio

(clinico, epidemiologico, viaggiatori internazionali, stile di vita o altro) 


\begin{tabular}{ccc}
\hline & $\mathbf{N}=\mathbf{4 3 2}$ & $\%$ \\
\hline Genere & & \\
Maschile & 292 & 67,6 \\
\hline Femminile & 140 & 32,4 \\
\hline Nazionalità & & \\
\hline Italiana & 354 & 81,9 \\
Straniera & 78 & 18,1 \\
\hline Età & & \\
$0-9$ & 2 & 0,5 \\
$10-19$ & 3 & 0,7 \\
$20-29$ & 23 & 5,5 \\
$30-39$ & 45 & 10,8 \\
$40-49$ & 103 & 24,8 \\
$50-59$ & 169 & 40,7 \\
$60-69$ & 59 & 14,2 \\
$70-79$ & 10 & 2,4 \\
$>80$ & 1 & 0,2 \\
\hline & & \\
\hline
\end{tabular}

Tabella 5. Pazienti con infezione da HIV presi in carico dal centro vaccinale.

inserire nuovi pazienti, effettuare statistiche. Ciascun soggetto inserito è identificato da un codice che ne garantisce l'anonimato.

Nell'ambito del LIV in HIV, è stato avviato il processo di collegamento automatico tra la piattaforma già esistente, i singoli sistemi demografici ospedalieri e le anagrafi vaccinali informatizzate, con l'obiettivo di individuare i soggetti suscettibili, calcolare il numero di dosi somministrate e conoscere il denominatore per le coperture vaccinali (33).

Inoltre, grazie alla capacità del sistema di ricevere dati di laboratorio, è possibile, per ciascun paziente, visualizzare i risultati delle indagini sierologiche effettuate, utili sia per lo screening prevaccinale (catch-up dei non vaccinati) sia per valutare la risposta alla vaccinazione e l'eventuale pianificazione di dosi di richiamo.

\section{L'esperienza dell'area metropolitana genovese}

Come esempio di contestualizzazione territoriale del LIV in HIV, secondo un percorso multidisciplinare integrato, viene di seguito riportata l'esperienza maturata presso l'ambulatorio di Prevenzione delle Malattie Trasmissibili, Vaccinazioni e
Sperimentazioni Cliniche dell'U.O. Igiene dell'Ospedale Policlinico San Martino- IST e gli ambulatori di Clinica di Malattie Infettive dell'Ospedale Policlinico San Martino- IST e dell'E.O. Ospedali Galliera, centri di riferimento per l'area metropolitana genovese.

Durante le visite routinarie di follow-up presso l'ambulatorio di Malattie Infettive, i soggetti suscettibili vengono individuati ed incoraggiati a raggiungere il centro vaccinale per lo screening sierologico e le vaccinazioni del caso. Per favorire l'adesione alla vaccinazione, l'ambulatorio vaccinale ha parallelamente svolto anche attività de-localizzata presso l'ambulatorio di Malattie Infettive dell'E.O. Ospedali Galliera, avviando una collaborazione che ha portato il servizio direttamente al paziente, presso il centro di diagnosi e cura, al quale afferisce per la gestione clinica e terapeutica.

Presso il centro vaccinale viene effettuata la valutazione dello stato sierologico e vaccinale accedendo alla piattaforma web. Ai pazienti vengono proposte le vaccinazioni raccomandate sulla base del calendario della Regione Liguria, con la possibilità di garantire la gratuità dell'offerta. Sulla base dell'indicazione del medico specialista di riferimento e delle caratteristiche individuali (co-morbosità, comportamenti a rischio) vengono scelte le vaccinazioni prioritarie e organizzati gli appuntamenti per le sedute vaccinali, tracciando pertanto un percorso individualizzato. L'integrazione con gli altri specialisti è facilitata dalla registrazione delle vaccinazioni eseguite sul portale web Ligurian HIV Network, attraverso I'interfaccia dedicata alle sedute vaccinali.

Da gennaio 2014 a luglio 2017, sono stati presi in carico dal centro vaccinale 432 pazienti con infezione da HIV: di questi, 374 (86,6\%) provengono dalla Clinica di Malattie infettive dell'Ospedale Policlinico San Martino-IST, 58 (13,4\%) dell’E.O. Ospedali Galliera. La Tabella 5 riporta le caratteristiche della popolazione in studio.

Dall'1/01/2014 all'11/07/2017 sono stati registrati 1547 accessi da riferirsi a pazienti con infezione da HIV, su un totale di 12.800.

L'incremento di questi, a partire dall'attivazione del nuovo percorso assistenziale (ottobre 2015), è risultato statisticamente significativo $(p<0,05)$, passando dal 5.2\% (191/3677) nel periodo gennaio 2014-ottobre 2015, al 14,86\% (1356/9123) 


\begin{tabular}{ccc}
\hline Vaccino & \multicolumn{2}{c}{ Dosi } \\
& N & $\%$ \\
dT & 1 & 0,06 \\
dTpa & 48 & 3,11 \\
dTpalPV & 12 & 0,77 \\
Flu & 322 & 20,87 \\
HAV & 68 & 4,41 \\
HBV & 216 & 14,00 \\
HBV+HAV & 101 & 6,55 \\
HIB & 82 & 5,31 \\
HPV4 & 49 & 3,17 \\
MenACWY & 148 & 9,59 \\
MenB & 158 & 10,24 \\
\hline MenC & 1 & 0,06 \\
MPR & 8 & 0,52 \\
PCV13 & 241 & 15,62 \\
PPV23 & 79 & 5,12 \\
VZV & 9 & 0,58 \\
Totale & 1543 & 100 \\
\hline
\end{tabular}

Tabella 6. Dosi somministrate in pazienti con infezione da HIV, stratificate per tipologia di vaccino (periodo 2014-2017).

\section{Legenda:}

dT: difterite-tetano; $\mathbf{~ I T p a : ~ d i f t e r i t e , t e t a n o , p e r t o s s e ; ~} \mathbf{d T p a I P V}$ : difterite,tetano,pertosse,polio; Flu: influenza; HAV: epatite A; HBV: epatite B; HBV+HAV: combinato epatite A ed epatite b; HIB: hemophilus di tipo B; HPV: papilloma virus quadrivalente; MenACWY: meningococco coniugato tetravalente; $\mathbf{M e n B}$ : meningococco gruppo B; MenC: meningococco gruppo C; MPR: morbillo, parotite,rosolia; PCV13: pneumococco coniugato 13-valente; PPV23: pneumococco polisaccaridico 23-valente; VZV: varicella.

nel periodo novembre 2015-luglio 2017 (dato non mostrato).

Nella Tabella 6 è indicata la distribuzione dei preparati vaccinali e il numero di dosi per preparato. La Figura 1 descrive gli accessi giornalieri dei soli pazienti con infezione da HIV. I picchi sono attribuibili a giornate dedicate principalmente alla vaccinazione antinfluenzale stagionale (stagione 2015-2016 e 2016-2017).

La Figura 2 indica il numero di pazienti che hanno ricevuto almeno una vaccinazione nel corso dell'anno. Si sottolinea come ogni anno ci sia stato un incremento di quasi il 50\% dell'utenza suddetta; questo dato sembra confermarsi anche nei primi sei mesi del 2017.

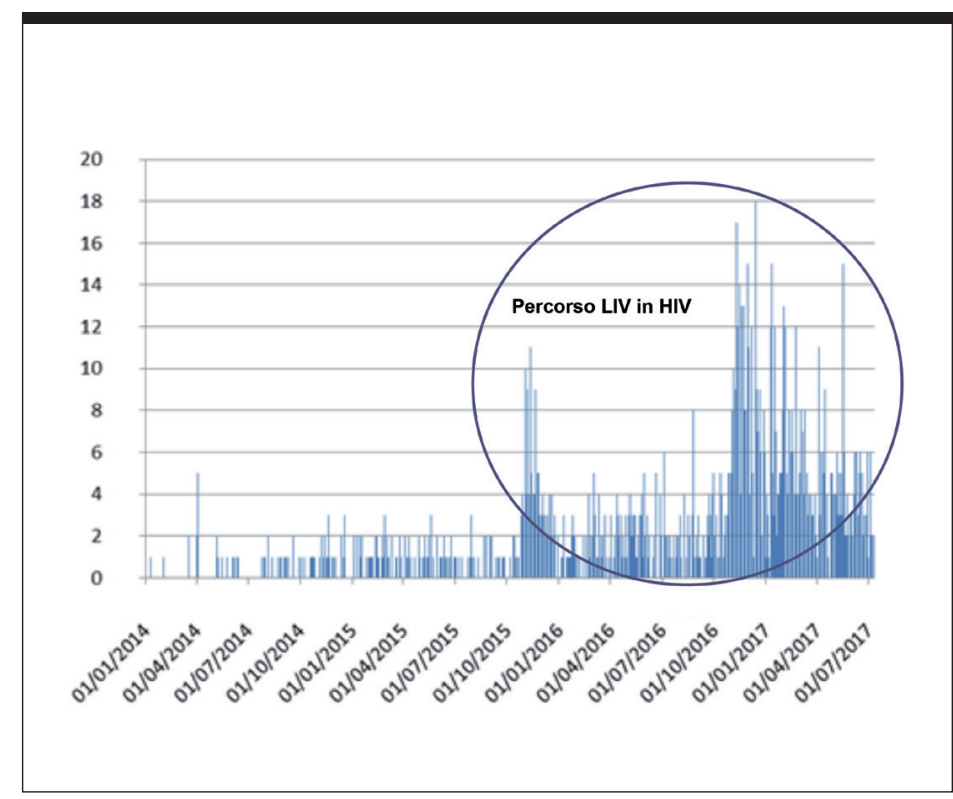

Figura 1. Accessi giornalieri all'ambulatorio vaccinale dei pazienti con infezione da HIV (periodo 2014-2017).

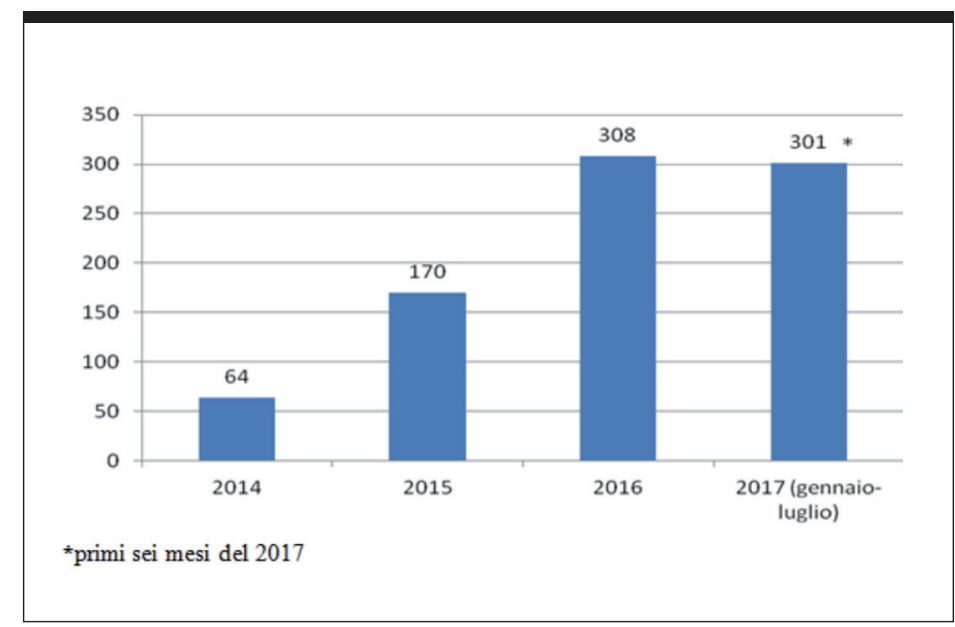

Figura 2. Pazienti con infezione da HIV che hanno effettuato almeno una vaccinazione nel corso dell'anno, presso l'ambulatorio UO Igiene (periodo 2014-2017).

\section{Conclusioni}

Il percorso di vaccinazione introdotto dal programma LIV in HIV rappresenta la prima esperienza nella nostra regione di attivazione di strategie selettive per l'implementazione e la promozione delle vaccinazioni nei soggetti con infezione da HIV. Gli strumenti che hanno permesso di avviare questo programma sono stati l'offerta attiva e gratuita delle vaccinazioni e la gestione informatizzata della 


\section{articolo originale}

popolazione target, consentendo una reale integrazione tra i dati clinici, vaccinali e di laboratorio.

La peculiarità inoltre di potersi avvalere di un servizio vaccinale, ad acceso libero, presente all'interno della stessa struttura ospedaliera, che favorisca paziente e medico nell'organizzazione delle sedute vaccinali, contestualmente ai follow-up infettivologici, ha sicuramente facilitato l'adesione dei pazienti. Altre modalità di raggiungimento dei pazienti, attraverso un "ambulatorio vaccinale mobile" per raggiungerli di- rettamente negli ambulatori dei centri di riferimento per la cura e la terapia, sono strategie utili, ma più difficili da realizzare dal punto di vista organizzativo e in contesti con scarse risorse. II percorso dedicato e integrato di immunizzazione del paziente con infezione da HIV in Liguria rappresenta una nuova sfida per la tutela della salute di questo peculiare gruppo a rischio, per il quale la gestione della cronicità necessita di percorsi definiti e ampiamente condivisi con tutti gli specialisti coinvolti.

\section{BIBLIOGRAFIA}

1. Crum-Cianflone NF, Grandits G, Echols S, et al. Trends and Causes of Hospitalizations Among HIV-Infected Persons During the Late HAART Era: What is The Impact of CD4 Counts and HAART Use? JAIDS. 2010; 54: 248-57.

2. British HIV Association Guidelines on the use of Vaccines in HIV-positive adults 2015.http://www.bhiva.org/documents/Guidelines/ Vaccination/2015-Vaccination-Guidelines.pdf

3. Rubin LG, Levin MJ, Ljungman P, et al. 2013 IDSA Clinical Practice Guideline for Vaccination of the Immunocompromised Host. Clin Inf Dis 2014; 58: e44-100.

4. Kim DK, Riley LE, Harriman KH, Hunter P, Bridges CB. Advisory Committee on Immunization Practices Recommended Immunization Schedule for Adults Aged 19 Years or Older - United States, 2017. MMWR Morb Mortal Wkly Rep 2017; 66: 136-8.

5. Tedaldi EM, Baker RK, Moorman AC, et al. HIV Outpatient Study (HOPS) Hepatitis A and B vaccination practices for ambulatory patients infected with HIV. Clin Infect Dis. 2004; 38: 1478-84.

6. Mohseni-Zadeh M, Rey D, Batard ML, et al. Inadequate vaccination coverage in a French cohort of HIV positive patients. Med Mal Infect 2010; 40: 683-90.

7. Zinna S, Bamford A, Cunnington A, et al. Immunization status of children with HIV: failure to protect a vulnerable population. HIV Med 2011; 12: 447-8.

8. Pandolfi E, Carloni E, Marino MG, et al. Immunization coverage and timeliness of vaccination in Italian children with chronic diseases. Vaccine 2012; 30: 5172-8.

9. Barre-Sinoussi F, Ross AL, Delfraissy JF. Past, present and future: 30 years of HIV research. Nat Rev Microbiol 2013; 11: 877-83.

10. Dalgleish AG, Beverley PC, Clapham PR, Crawford DH, Greaves MF, Weiss RA. The CD4 (T4) antigen is an essential component of the receptor for the AIDS retrovirus. Nature 1984; 312: 763-7.

11. Nicolini LA, Giacobbe DR, Di Biagio A, Viscoli C. Insights on common vaccinations in HIV-infection: efficacy and safety. J Prev Med Hyg 2015; 56: E28-E32.

12. Overton ET. An overview of vaccinations in HIV. Curr HIV/AIDS Rep 2007; 4:105-13.

13. Veiga AP, Casseb J, Duarte AJ. Humoral response to hepatitis B vaccination and its relationship with $T$ CD45RA+ (naïve) and CD45RO+ (memory) subsets in HIV-1-infected subjects. Vaccine 2006; 24: 7124-8.

14. Kourkounti S, Papaizos V, Leuow K, Kordosis T, Antoniou C. Hepatitis A vaccination and immunological parameters in HIV-infected patients. Viral Immunol 2013; 26: 357-63.

15. Parmigiani A, Alcaide ML, Freguja R, et al. Impaired antibody response to influenza vaccine in HIV-infected and uninfected aging women is associated with immune activation and inflammation. PLoS One 2013; 8: e79816.

16. Fuller JD, Craven DE, Steger KA, Cox N, Heeren TC, Chernoff D. Influenza vaccination of human immunodeficiency virus (HIV)infected adults: impact on plasma levels of HIV type 1 RNA and determinants of antibody response. Clin Infect Dis 1999; 28: 541-7.

17. Menson EN, Mellado MJ, Bamford A, et al. Paediatric European Network for Treatment of AIDS (PENTA) Vaccines Group; PENTA Steering Committee; Children's HIV Association (CHIVA). Guidance on vaccination of HIV-infected children in Europe. HIV Med 2012; 13: 333-6.

18. Kernéis S, Launay O, Turbelin C, Batteux F, Hanslik T, Boëlle PY. Long-term immune responses to vaccination in HIV-infected patients: a systematic review and meta-analysis. Clin Infect Dis. 2014; 58: 1130-9.

19. Crum-Cianflone NF, Sullivan E. Vaccinations for the HIV-Infected Adult: A Review of the Current Recommendations, Part I-II. Infect Dis Ther 2017; ahead of print; doi: 10.1007/s40121-017-0166-x

20. Geretti AM, Doyle T. Immunization for HIV-positive individuals. Curr Opin Infect Dis 2010; 23: 32-8.

21. Crane HM, Dhanireddy S, Kim HN, et al. Optimal timing of routine vaccination in HIV-infected persons. Curr HIV/AIDS Rep 2009; 6: 93-9.

22. Crum-Cianflone NF, Wallace MR. Vaccination in HIV-infected adults. AIDS Patient Care STDS 2014; 28: 397-410. 
23. Sullivan PS, Hanson DL, Dworkin MS, Jones JL, Ward JW; Adult and Adolescent Spectrum of HIV Disease Investigators. Effect of influenza vaccination on disease progression among HIV-infected persons. AIDS 2000;14: 2781-5.

24. Panel on Opportunistic Infections in HIV-Infected Adults and Adolescents. Guidelines for the prevention and treatment of opportunistic infections in HIV-infected adults and adolescents: recommendations from the Centers for Disease Control and Prevention, the National Institutes of Health, and the HIV Medicine Association of the Infectious Diseases Society of America. https://aidsinfo.nih. gov/contentfiles/Ivguidelines/adult_OI.pdf. Ultimo accesso in data 31 Agosto 2017

25. Aberg JA, Gallant JE, Ghanem KG, Emmanuel P, Zingman BS, Horberg MA. Primary care guidelines for the management of persons infected with HIV: 2013 update by the HIV medicine association of the Infectious Diseases Society of America. Clin Infect Dis 2014; 58: e1-34.

26. European AIDS Clinical Society (EACS) guidelines for the treatment of HIV. Version 8.2. January 2017. Link: http://www.eacsociety. org/guidelines/eacs-guideines/eacs-guidelines. html. (ultimo accesso in data 31 agosto 2017)

27. Linee Guida italiane sull'utilizzo dei farmaci antiretrovirali e sulla gestione diagnostico-clinica delle persone coninfezione da HIV-1, edizione 22 novembre 2016. Available at: http://www.salute.gov.it/imgs/C_17_pubblicazioni_2545_allegato.pdf. (ultimo accesso in data 31 agosto 2017)

28. Conferenza Stato-Regioni il 19 gennaio 2017. Piano Nazionale Prevenzione Vaccinale 2017-2019 (PNPV). Gazzetta Ufficiale del 18 febbraio 2017. Available at: http://www.salute.gov.it/imgs/C_17_pubblicazioni_2571_allegato.pdf (ultimo accesso in data 31 agosto 2017

29. Deliberazione della Giunta Regionale n. 1701 del 22/12/2014 (oggetto: Piano Regionale Prevenzione Vaccinale, aggiornamento

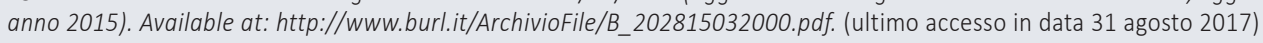

30. Deliberazione della Giunta Regionale 30.11.2015 N. 1314. Integrazione del Piano Regionale Prevenzione Vaccinale con il calendario vaccinale per i soggetti con infezione da HIV. Regione Liguria. Available at: http://www.burl.it/ArchivioFile/B_217415512000.pdf (ultimo accesso in data 31 agosto 2017)

31. Giuliano AR, Isaacs-Soriano K, Torres BN, Abrahamsen M, Ingles DJ, Sirak BA, Quiterio M, Lazcano-Ponce E. Immunogenicity and safety of Gardasil among mid-adult aged men (27-45 years)--The MAM Study. Vaccine 2015; 33: 5640-6.

32. Fraccaro $P$, Dentone $C$, Fenoglio D, Giacomini M. Multicentre clinical trials' data management: a hybrid solution to exploit the strengths of electronic data capture and electronic health records systems. Informatics Heal Soc Care 2013; 38: 313-29.

33. Giannini B, Gazzarata R, Sticchi L, Giacomini M. A SOA-Based Solution to Monitor Vaccination Coverage Among HIV-Infected Patients in Liguria. Stud Health Technol Inform 2016; 228: 327-31. 